Methods Design and setting: A pragmatic randomised controlled trial in a level III NICU including infants born at 32 weeks gestation and their parents. Exclusion criteria were infant major congenital abnormality and maternal low level of fluency in English. Intervention: comprised reflective interview, observation of infant cues and Video Interaction Guidance (VIG). The primary outcome, maternal sensitivity during playful interaction with her infant was measured by the Child Adult Relationship Evaluation (CARE-Index). Secondary outcomes were infant social-emotional problems measured by the Ages and Stages Questionnaire-Social-Emotional version (ASQSE) and self-reported parental mental health. Modification of the effect of the programme on the primary outcome, maternal sensitivity, was explored using general linear model univariate analysis of sociodemographic, maternal and infant characteristics. Significant interactions are presented.

Results Eighty mothers and their preterm infants were randomized to the intervention and standard care groups. The groups were similar in baseline sociodemographic and perinatal characteristics, although more mothers in the intervention group had completed higher-level education and subsequent analyses were adjusted accordingly. At 12 months corrected age (CA) infants in the intervention group had fewer self-regulation problems at 12 months of age (ChiSquare $3.84, \mathrm{df}=1, \mathrm{p}=0.05$, partial eta squared $=0.07$ ) and infants whose mothers had received VIG had significantly fewer communication problems (Chi square $=20.41, \mathrm{df}=6$, $\mathrm{p}=0.002$, phi $=0.61$ ), however there was no statistically significant difference between the intervention and standard care groups in maternal sensitivity during play at 9 months CA or measures of maternalmentalhealth. Fathers in the intervention group had lower depression scores at folllow up.There was modifying effect of maternal ethnicity $(B=4.179, C I=0.921-7.437, p=0.013)$; there was a significant difference in mean sensitivity of mothers of infants with IVH, (1. 85 points) $(\mathrm{CI}=0.083-3.614, \mathrm{p}=0.041)$ but there was no significant interaction with group assignment.

Conclusion This early attachment focused intervention integrating VIG for mothers and their very preterm infants had significant positive effects on infant social-emotional problems at 12 months CA.Maternal ethnicity modified the intervention effect

\section{GP82 PREVALANCE RATES FOR AVOIDANT RESTRICTIVE FOOD INTAKE DISORDER (ARFID) IN TERTITARY FEEDING CLINIC IN UK}

Lucy Spicer*, Katy Strudwick, Veronica Kelly. Evelina London Children's Hospital, London, UK

10.1136/archdischild-2019-epa.148

We present the prevalence rates of Avoidant Restrictive Food Intake Disorder (ARFID) in our tertiary feeding service. ARFID is a relatively recent DSM -V diagnostic category that describes children with complex feeding disorders. Previous diagnoses of 'feeding disorders of infancy or early childhood' in the DSM-IV were limited and excluded children who maintained a healthy weight in the context of problematic eating behaviours. According to the DSM V in 2013 ARFID describes those children with an apparent lack of interest in food, food-avoidance based on its sensory characteristics and/or concerns about the aversive consequences of eating due to persistent failure to meet appropriate nutritional and/or energy needs are all symptomatic of ARFID.

The prevalence rates of ARFID are currently unknown. In specialist psychiatric and medical settings it is estimated to be between $5-13 \%$. The clinical characteristics of children with complex feeding difficulties are currently poorly described in the literature, making it difficult to identify and plan necessary services.

Our service is a tertiary multidisciplinary feeding service that receives on average 250 referrals per annum. Of those referrals, 150 are referred to $\mathrm{r}$ the Complex Feeding Clinic and the others are for medical, dysphagia or videofluroscopy clinics. The Complex Feeding Service is formed of a Consultant Paediatrician, Psychologist, Occupational Therapist, Dietictian and Speech and Language Therapist. We accept referrals for children both locally and regionally, on the provision that there is a named local professional to liaise with. Our referral criteria includes children up to 18 years old with complex feeding difficulties which may cause psychosocial or nutritional impairment. Such feeding difficulties may be related to: an underlying ASD diagnosis, a developmental feeding disorder, medical disorder, sensory selectivity and/or anxiety around foods. The children who attend our clinic may be fed by enteral means or have a selective eating pattern (not otherwise stated).

We chart the emergence of this diagnosis within our cohort of patients and track its increasing use since it was included in the DSM V in 2013. We also analyse the following characteristics within the cohort of children receiving this diagnosis: gender, age, with/without a diagnosis of ASD or other co morbid neurodevelopmental issues, nutritional adequacy, diet range and fed by enteral means i.e. NG/PEG.

\section{GP83 FOETAL VALPROATE SYNDROME, DEVELOPING A NEW SERVICE}

Jennifer Geraghty, Anitha Sokay, Attia Kalim*. Our Lady's Childre's Hospital Crumlin, Dublin, Ireland

\subsection{6/archdischild-2019-epa.149}

Introduction A Paediatric valproate clinic at Our Lady's Children's Hospital Crumlin was established to assess those children who were exposed to sodium valproate antenatally in Ireland and to determine if this exposure is the contributing factor to their developmental delay, autism or skeletal malformations.

Antiepileptic drugs (AEDs) have been associated with a two to three fold increase in major malformations in children exposed to AEDs in-utero, compared to the general population. ${ }^{1-2}$

Children exposed to sodium valproate compared to other AEDs had the highest level of risk of a malformation at $10.93 \%$. $^{3}$

Methods A national referral service was established via the HSE Valproate Support Team. GP were advised to refer all children less than sixteen years of age exposed to valproate antenatally. Thirteen children from six families were reviewed in the first six weeks. Background diagnosis, developmental history, maternal valproate history and full clinical examination findings were noted. Data was collected and recorded via a proforma. 